Bio-grafía Escritos sobre la Biología y su Enseñanza.

Edición Extra-Ordinaria.

Memorias del I Congreso Nacional de Investigación en Enseñanza de la

Biología. VI Encuentro Nacional de Investigación en Enseñanza de la Biología y la Educación Ambiental. ISSN 2027 1034. P. p. 342-350.

\title{
EL ORIGEN DEL CONCEPTO ECOSISTEMA ${ }^{1}$
}

\section{THE ORIGIN OF THE ECOSYSTEM CONCEPT}

\section{POR: María Eugenia Rincón ${ }^{2}$}

\section{Resumen}

El ecosistema ha sido un concepto clave en el desarrollo de la ecología moderna; sin embargo, hoy en día ha sido muy mal interpretado y tergiversado. El desarrollo del concepto ecosistema coincide con un periodo de cambios en la manera de enfocar este término, desde el holismo Clementsiano, el cual predominó en la primera mitad del siglo XX, hasta el reduccionismo dominante hoy en día. Este concepto surge como un argumento teórico, en el contexto de la teoría de la sucesión ecológica, como una clara refutación de Tansley a la ontología Clementsiana. El propósito de este trabajo es presentar una revisión sobre el origen del concepto ecosistema.

Palabras clave: Ecosistema, sucesión ecológica, holismo, individualismo.

\section{Abstract}

The ecosystem has been a key concept in the development of modern ecology. However, today it is widely misunderstood and misused. The development of the ecosystem concept coincides with a period of change in the approach to this term, from Clementsian holism, which predominated in the first half of the twentieth century, to the reductionism dominant today. This concept emerges as a theoretical argument in the context of the theory of ecological succession as a clear Tansley's refutation of Clementsian ontology. The purpose of this paper is to present a review of the origin of the ecosystem concept.

Keywords: ecosystem, ecological succession, holism, individualism.

\section{INTRODUCCIÓN}

Desde una perspectiva conceptual, el análisis del ecosistema tiene una variedad de significados: como idea filosófica, este fomenta la investigación y aplicación del concepto en estudios ambientales relacionados con la

\footnotetext{
${ }^{1}$ Este documento hace parte de los desarrollos de la tesis Doctoral "Conocimiento didáctico del contenido disciplinar del formador de formadores de biología sobre el concepto ecosistema". Doctorado interinstitucional en Educación. Universidad Pedagógica Nacional. Grupo de Investigación Conocimiento Profesional del Profesor de Ciencias.
}

${ }^{2}$ Docente Departamento de Biología. Universidad Pedagógica Nacional. 
Memorias del I Congreso Nacional de Investigación en Enseñanza de la

Biología. VI Encuentro Nacional de Investigación en Enseñanza de la Biología y la Educación Ambiental. ISSN 2027 1034. P. P. 342-350.

conservación y manejo del ambiente; como un concepto que identifica un objeto físico (en el sentido de Tansley), este es el tema de muchos estudios científicos y como un paradigma científico, este estructura la organización científica y la investigación (Golley, 1993). Con este uso tan amplio, no es de extrañar que este concepto haya sido malinterpretado y cuestionado por muchos ecólogos.

El concepto de ecosistema es particularmente adecuado en los programas de ecología, para la introducción de la historia de la ciencia y específicamente de la ecología, dado que abrió el camino para el debate de dos paradigmas en ecología: el holismo y el reduccionismo o individualismo. La clásica dicotomía entre holismo y reduccionismo constituye un marco adecuado desde el cual abordar el desarrollo histórico del concepto. Los holistas concentran su atención en cambios en la diversidad, la productividad, la biomasa y otras características del ecosistema, así como en la direccionalidad y la predictibilidad de las trayectorias sucesionales que conducen a un clímax único. Los reduccionistas enfatizan las perturbaciones, los fenómenos estocásticos, las historias de vida e interacciones entre especies, convencidos de que la sucesión supone la consecuencia impredecible de la interacción de cada una de las especies con su entorno biótico y abiótico (Glen- Lewin, Peet y Veblen, 1992). Sin embargo, desde una perspectiva contemporánea las dos aproximaciones se pueden integrar.

A pesar de que investigaciones recientes en la educación científica han argumentado la importancia de la historia y la filosofía de la ciencia para la enseñanza de la ciencia (Rodriguez y Niaz, 2002), podría afirmarse que la ausencia de una perspectiva histórica de los conocimientos que se enseñan constituye un elemento del común denominador en la escuela.

En relación con la enseñanza de la ecología, Golley (1993), señala que hay pocos estudios históricos de la ecología, por lo que se enseña esta disciplina como si no tuviera historia. El resalta el valor del enfoque histórico en su enseñanza más allá de centrar la atención en un concepto fundamental. Además, según Schwab (1974), la perspectiva histórica es importante para entender en el caso de una disciplina no sólo los detalles experimentales, sino también el principio heurístico que subyace en los resultados experimentales.

Teniendo en cuenta esta perspectiva, es importante analizar cómo se llevó a cabo el origen del concepto ecosistema y que implicaciones tiene en los desarrollos del concepto y de la ecología en general.

\section{ORIGEN DEL CONCEPTO ECOSISTEMA}

Desde los siglos XVIII y XIX los naturalistas empezaron a preocuparse por conocer formalmente los patrones de distribución de los organismos, tratando 
Memorias del I Congreso Nacional de Investigación en Enseñanza de la

Biología. VI Encuentro Nacional de Investigación en Enseñanza de la Biología y la Educación Ambiental. ISSN 2027 1034. P. p. 342-350.

de entender cómo se formaban y mantenían dichos patrones por la interacción de la biota con el ambiente físico. Sin embargo, su visión de las comunidades naturales era espacial y estática, debido a que no se consideraba que estas cambiaban en el tiempo. Fue hacia comienzos del siglo XX que se empezaron a considerar aspectos temporales en la distribución de los organismos.

A continuación se presenta de manera general, como el desarrollo de la teoría de la sucesión ecológica planteada por el ecólogo Frederick Clement en 1916 y publicada en Plant Succesion: An analysis of the Development of Vegetation, constituyó el marco contextual desde donde surgió el concepto de ecosistema. Clements equiparó a la sucesión con el desarrollo de un Superorganismo: "el organismo nace sobre un campo abandonado, con las plantas invasoras presentes en el sitio, se desarrolla y eventualmente llega a la madurez...Además de esto, cada formación climax es capaz de reproducirse a sí misma, repitiendo con esencial fidelidad los estadios de su desarrollo (Clements 1905:199, citado en Clements, 1916).

La teoría sucesional clásica adhería al paradigma de equilibrio, al asumir que toda comunidad progresaría invariablemente a una composición estable y a un estado final único de equilibrio con el clima regional, que se denomino "Climax climático". Dicha teoría, era además determinística al postular que el desarrollo del climax era tan ordenado y predecible como el desarrollo vital de un organismo.

Este concepto del superorganismo, planteado para la comunidad vegetal, tuvo gran influencia en otras áreas de la ecología y presento una amplia difusión entre los fundadores de la ecología animal y la limnología.

Esta visión supeorganismica de las comunidades, contrastó fuertemente con la idea individualista de Gleason quien en 1926, propuso que las comunidades estaban conformadas por poblaciones con arreglos aleatorios, producto de los patrones de distribución de cada especie (Gleason 1939). Según este planteamiento, cada especie tiene patrones individuales de distribución y las comunidades son simplemente el resultado de la superposición de varias poblaciones con una distribución común.

En todo este periodo, dominó la idea de que los organismos eran la parte esencial de las comunidades. No fue sino hasta 1935, que el botánico inglés Arthur George Tansley propuso el término ecosistema por primera vez, en un artículo titulado "The use and Abuse of Vegetational Concepts and Terms" en la revista Ecology. 
Memorias del I Congreso Nacional de Investigación en Enseñanza de la

Biología. VI Encuentro Nacional de Investigación en Enseñanza de la Biología y la Educación Ambiental. ISSN 2027 1034. P. p. 342-350.

Sin embargo, el estímulo inmediato que tuvo Tansley para esta publicación, fue la aparición de cuatro artículos publicados por el ecólogo sudafricano John Philips en (1931, 1934, 1935a y 1935b) en The Journal of ecology, relacionados con la comunidad biótica, sucesión, desarrollo y el climax y el organismo complejo, respectivamente. En el tratado final Pillips, estableció una conexión entre el pensamiento de Clements y la filosofía social holística de Jan Christian Smuts3 en defensa del concepto de la comunidad como un organismo complejo.

Básicamente, Tansley presento este concepto como una refutación de la ontología Clementsiana, el afirmo: "Personalmente he dado mis razones para rechazar los términos "organismo complejo" y comunidad biótica. El término bioma primeramente propuesto por Clements para el complejo integro de organismos que habitan una cierta región, es inobjetable y conveniente para ciertos propósitos; pero en mi opinión, el concepto fundamental corresponde al sistema completo (en el sentido físico), que incluye no solamente al organismocomplejo, sino también al complejo total de factores físicos que constituyen lo que denominamos el ambiente del bioma -los factores del hábitat en el más amplio sentido. Si bien los organismos constituyen nuestro interés principal, no podemos separarlos de su ambiente especial, ya que con el conforman un sistema físico... representan las unidades básicas de la naturaleza... En cada sistema existe intercambio constante del más variado tipo, no solo entre los organismos, sino entre la parte orgánica e inorgánica Estos ecosistemas, como nosotros podemos denominarlos son de las más diversas clases y tamaños (1935: 299).

Así, el concepto de ecosistema de Tansley identifico un sistema que era: 1 . Un elemento en una jerarquía de sistemas físicos desde el universo hasta el átomo, 2. El sistema básico de la ecología, y 3. Compuesto tanto de organismos como del ambiente físico. Desde su origen hasta el presente, el ecosistema de Tansley ha constituido un concepto clave de la ecología.

En relación con el concepto del organismo complejo o superorganismo, Tansley (1935), argumento dos razones en contra de ese concepto: El primer

${ }^{3}$ El concepto de Holismo de Smuts se baso en un proceso de evolución de las moléculas físicas a los individuos y en última instancia a la mente y el valor. El enfoque tiene similaridad con el planteado por Pierre Teilhard de Chardin en su libro The Phenomenon of Man. Smuts enfatizo que el individuo era un todo y que el holismo era la fuerza que creaba los todos. Este concepto, es considerablemente diferente del concepto de holismo usado en la ecología moderna. 
Bio -grafía Escritos sobre la Biología y su Enseñanza.

Edición Extra-Ordinaria.

Memorias del I Congreso Nacional de Investigación en Enseñanza de la

Biología. VI Encuentro Nacional de Investigación en Enseñanza de la Biología y la Educación Ambiental. ISSN 2027 1034. P. p. 342-350.

argumento es que este es un concepto idealista que no es investigable usando métodos ecológicos de análisis. El segundo argumento es entre reduccionismo y holismo. El reduccionismo afirma que podemos entender la naturaleza de un fenómeno al reducirlo a sus partes, el reduccionismo continúa usando métodos convencionales para profundizar cada vez más en el análisis; en contraste el holista ha hecho poco progreso en crear reglas de ensamblaje. El holismo toma otro enfoque. El holismo de Smuts ha sido descrito, pero el holismo puede ser descrito en una forma menos idealista.

Teniendo en cuenta lo anterior, se puede argumentar que el concepto de ecosistema se planteó como una solución a la discusión teórica que dividía a los ecólogos vegetales de esa época, en dos líneas opuestas. Un grupo enfatizaba en el significado individualista de las agrupaciones de vegetación y su inclusión en un sistema jerárquico de organización comunitaria. La otra aproximación entendía a la comunidad como un superorganismo. Parte de la motivación que tuvo Tansley de crear el concepto de ecosistema, fue el deseo de encontrar un puente que uniera estos dos puntos de vista en un enfoque ecológico.

Al igual que Tansley, el filósofo Alfred North Whitehead, reconoció la contradicción en la ciencia, la cual hacía hincapié en la primacía de los hechos y la interpretación individual de los hechos, pero sin embargo, tenía el gran propósito de descubrir los patrones y el orden en el mundo natural. De acuerdo a Whitehead (1949), este contraste entre una interpretación determinística y una relativista de eventos particulares, también como una generalización que lleva a una conclusión general basada en el pensamiento racional, constituía un problema general para la ciencia y la cultura. Mientras que estas contradicciones podrían evitarse dentro de las ciencias físicas y químicas debido a que las totalidades reconocidas por estos científicos eran físicas, el problema era central para la biología y las ciencias sociales (Golley, 1993). En ecología el contraste era ilustrado por la teoría deterministica de la sucesión Clementsiana, en contraste al enfoque relativista o individualista defendido por Henry Gleason.

En general, el concepto de ecosistema planteado por Tansley, fue un concepto físico, que enfatizó en la interacción de los componentes físicos, químicos y biológicos que actuaban juntos para formar un ecosistema, el cual a su vez formaba parte de una jerarquía de sistemas físicos desde el universo hasta el átomo. Según Keller y Golley (2000), el lenguaje que usó Tansley en su concepto de ecosistema se derivo de las ideas científicas y filosóficas de comienzos del siglo XX, principalmente retomando los conceptos de sistema y de equilibrio físico. A diferencia de los ecólogos de la comunidad o el organismo complejo, el ecosistema de Tansley estaba integrado por el 
Memorias del I Congreso Nacional de Investigación en Enseñanza de la

Biología. VI Encuentro Nacional de Investigación en Enseñanza de la Biología y la Educación Ambiental. ISSN 2027 1034. P. P. 342-350.

ambiente físico químico y la biota entera. Estos conceptos estaban relacionados y juntos representaron un aspecto diferente de las ideas más amplias que fueron derivadas tanto de la interpretación científica de la naturaleza y el concepto holístico de la filosofía europea occidental.

Los argumentos planteados por Tansley son problemáticos, desde mi punto de vista, por el énfasis que él le otorgó al concepto de ecosistema, desde una perspectiva física. Sus afirmaciones acerca de que los ecosistemas corresponden a un nivel de una jerarquía de sistemas físicos que van desde el planeta tierra a los átomos, se opone a la perspectiva ecológica acerca de la noción de sistema. El concepto físico de una entidad es un objeto aislado, material, que se explica a través de la interacción estructural de sus partes. A diferencia de los sistemas físicos, en los sistemas ecológicos los procesos son estocásticos y están interconectados con procesos externos al sistema. Ellos son sistemas abiertos y su organización se deriva tanto de sus conexiones con el entorno como con sus interacciones entre las partes del sistema. De hecho, desde una perspectiva moderna, se ha producido un importante cambio de paradigma en la visión de los ecólogos acerca de la naturaleza del cambio en los ecosistemas, el paradigma del estado estable y el equilibrio biológico fue reemplazado por el del no equilibrio.

Tansley uso la idea de sistema frecuentemente en sus escritos. Esta servía a su preocupación acerca de la organización de la naturaleza. La ciencia de los sistemas fue una aplicación técnica de la filosofía holística materialista. Independientemente del medio que estudiaran (dulceacuícola, marino o terrestre), los biólogos de principios del siglo XX comenzaron a tener la idea de que la naturaleza funciona como un sistema.

La propuesta de Tansley, se propagó rápidamente durante finales de los años treinta y fue adoptada principalmente en estudios de limnología. Se considera que Raymond Lindeman, fue el primero en implementar el concepto de Tansley en términos cuantitativos, para definir el sistema y describir su comportamiento dinámico. La obra de Lindeman es sorprendentemente actual, pues el introdujo la mayoría de los conceptos de la energética ecológica moderna. El uso del concepto de energía por Lindeman para expresar las relaciones entre los niveles tróficos, de nuevo relacionaba la ecología con la teoría de la termodinámica en física. Igualmente, el reconocido limnologo, Thienemann también visualizó el sistema como un organismo de un orden superior, de la misma forma que Clements entendía a la vegetación como un organismo complejo.

Se podría afirmar que las ideas sucesionales clásicas de Clements, dominaron el pensamiento ecológico durante la primera mitad del siglo XX a pesar de 
Memorias del I Congreso Nacional de Investigación en Enseñanza de la

Biología. VI Encuentro Nacional de Investigación en Enseñanza de la Biología y la Educación Ambiental. ISSN 2027 1034. P. p. 342-350.

tempranas y agudas críticas por parte de Gleason, quien las publico tres veces $(1917,1926,1927)$. Si bien Gleason fue inicialmente ignorado por la mayoría de los ecólogos, su fuerte desafío a los supuestos fundamentales de la teoría sucesional Clementsiana son hoy en día ampliamente aceptados (Glenn Lewin, Peet, y Veblen, 1992).

Colinvaux (1980), afirmo respecto al impacto de la perspectiva de Clements: "Esta teoría era tan emocionante que logro detener durante casi medio siglo el desarrollo de la ecología como disciplina realmente científica” (p. 617).

Según Keller y Golley (2000), los ecos de Clements aun repercuten en los proyectos de investigación ecológica. Ellos plantean que la hipótesis Gaia es básicamente el concepto del superorganismo Clementiano aplicado a la biosfera entera: "Esta elegante ontología teleológica fue convincente para la mayoría de los ecólogos. La teoría corroboro las observaciones de los ecólogos, suministro un esquema simple y determinístico de organizar las observaciones y fue predictiva" (p. 28).

El concepto de ecosistema ha sido criticado, por constituir una continuación de la idea del superorganismo planteado por Clements. El superorganismo fue una metáfora engañosa que atribuyo las propiedades del desarrollo de un organismo a la vegetación como una totalidad. Un problema con esta forma de razonamiento es que la comunidad vegetal se planteo como una abstracción distinta de la asociación vegetal y los parches de vegetación. Por lo que fue entonces posible unir dicha abstracción con el concepto idealista de Phillips.

Este fue el argumento presentado por Tansley, cuando el formulo el concepto de ecosistema como una alternativa al superorganismo. Que los ecólogos aún sigan confundiendo el superorganismo de Clements y el ecosistema da testimonio de la persistencia de estas dificultades conceptuales en los desarrollos de la ecología. Es irónico que el concepto de ecosistema haya sido criticado por ser superorganismico, cuando en realidad fue propuesto por Tansley justo como una alternativa al superorganismo.

\section{CONSIDERACIONES FINALES}

Esta reconstrucción preliminar acerca del origen del concepto ecosistema, muestra que el paradigma holista marco durante gran parte del siglo XX, los desarrollos conceptuales de la ecología y direcciono los estudios de la ecología de los ecosistemas durante varias décadas.

El concepto de ecosistema emergió como un argumento teórico, no fue el resultado, como en el caso de otros conceptos ecológicos de estudios técnicos y no se presentó como una síntesis de un campo de observaciones. Se puede 
Memorias del I Congreso Nacional de Investigación en Enseñanza de la

Biología. VI Encuentro Nacional de Investigación en Enseñanza de la Biología y la Educación Ambiental. ISSN 2027 1034. P. p. 342-350.

plantear que este concepto surgió como una solución a la discusión teórica que dividía a los ecólogos vegetales en dos líneas opuestas. Un grupo enfatizó el significado individual de las agrupaciones de la vegetación y su inclusión en un sistema jerárquico de organización comunitaria. La otra aproximación entendía a la comunidad como un superorganismo. Parte de la motivación de Tansley de proponer el concepto de ecosistema fue su deseo de encontrar un puente que uniera estos dos puntos de vista en un enfoque ecológico. Con el planteamiento de este concepto, Tansley inició una nueva dirección en los desarrollos de la ecología como disciplina, la Ecología de los ecosistemas.

Desde la perspectiva holista, la dinámica de los ecosistemas se asocia con un aumento de la estabilidad, la complejidad, la madurez y el climax del ecosistema. Esta forma de concebir el ecosistema, conlleva a una excesiva simplificación de sus procesos, pues estas ideas son de carácter determinista y contribuyen a restar valor científico a la ecología que se enseña en el aula y a reafirmar las ideas teleológicas de los estudiantes. Con base en lo anterior, se resalta la importancia de incorporar en la enseñanza de la ecología el enfoque histórico, de tal forma que ofrezca elementos que les permitan a los estudiantes: la comprensión del proceso histórico de producción de conocimiento ecológico, de la construcción teórico conceptual y de las estrategias metodológicas empleadas para ello, lo cual fomentara el interés de los estudiantes por la ecología y propiciara un mejor aprendizaje de la misma.

\section{AGRADECIMIENTOS}

Agradezco al Doctor Edgar Valbuena (Director de la tesis), por su valiosa colaboración y sugerencias para la realización de este trabajo. 
Edición Extra-Ordinaria.

Memorias del I Congreso Nacional de Investigación en Enseñanza de la

Biología. VI Encuentro Nacional de Investigación en Enseñanza de la Biología y la Educación Ambiental. ISSN 2027 1034. P. P. 342-350.

\section{BIBLIOGRAFIA}

- Clements, F. E. (1916). An Analysis of the Develeopment of Vegetation. Washington, D.C.: Carnegie Institution of Washington, Publication No 242, pp. 1-7.

- Colinvaux, P.A. (1980). Introducción a la ecología. Mexico: Editorial Limusa

- Gleason, H. A. 1939. The Individualistic Concept of Plant Association. American Midland Naturalist 21: 92- 110, pp. 92-107.

- Glenn-Lewin, D.C., R.K. Peet y T.T. Veblen, editors. 1992. Plant Succession: Theory and Prediction. Chapman and Hall, London, UK.

- Golley, F.B. (1993). A History of the Ecosystem Concept in Ecology: More than the sum of the Parts. United States of America: Yale University Press.

- Keller, D. R. y Golley, F. (2000). The philosophy of ecology. From science to synthesis. United States of America: The University of Georgia Press.

- Rodríguez, M. A. y Niaz M. (2002). How in spite of the Rhetoric, History of Chemistry has Been Ignored in Presenting Atomic Structure in Textbooks. Science \& Education, 11, 423- 441.

- Schwab, J.J.: 1974, 'The Concept of the Structure of a Discipline', In E.W. Eisner and E. Vallance (eds), Conflicting Conceptions of Curriculum, McCutchan, Berkeley, CA .

- Tansley, A. G. (1935). The Use and Abuse of Vegetational Concepts and Terms. Ecology, 16, (3), 284-307. 